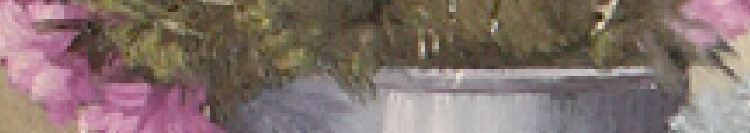

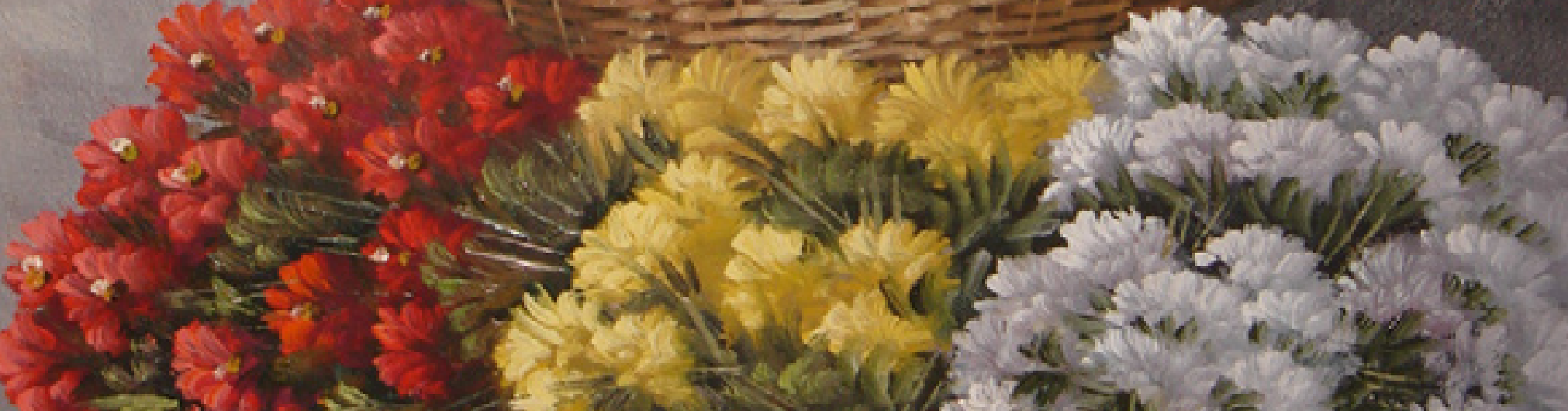
$+\frac{\pi}{4} \cos \frac{2}{2}$

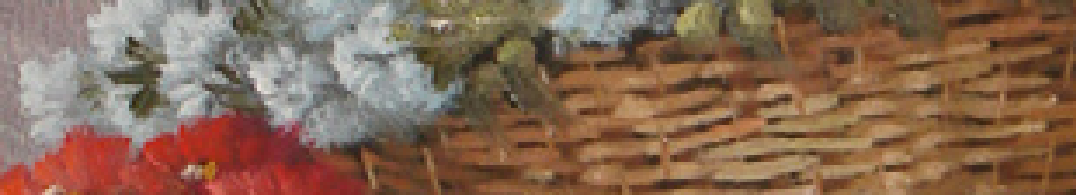

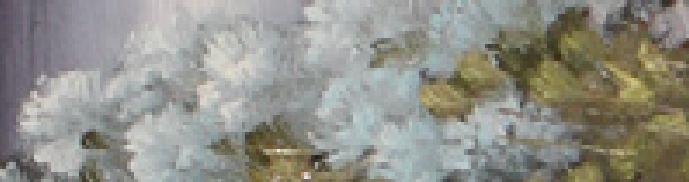

से दो $90=5$

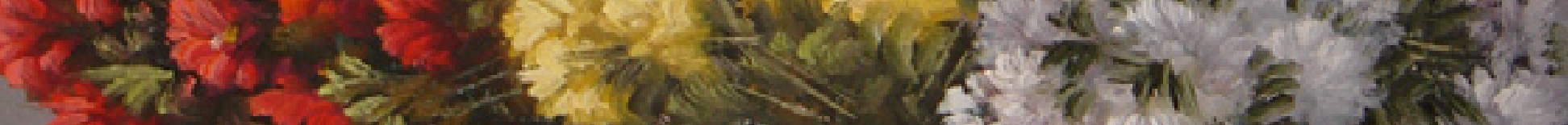

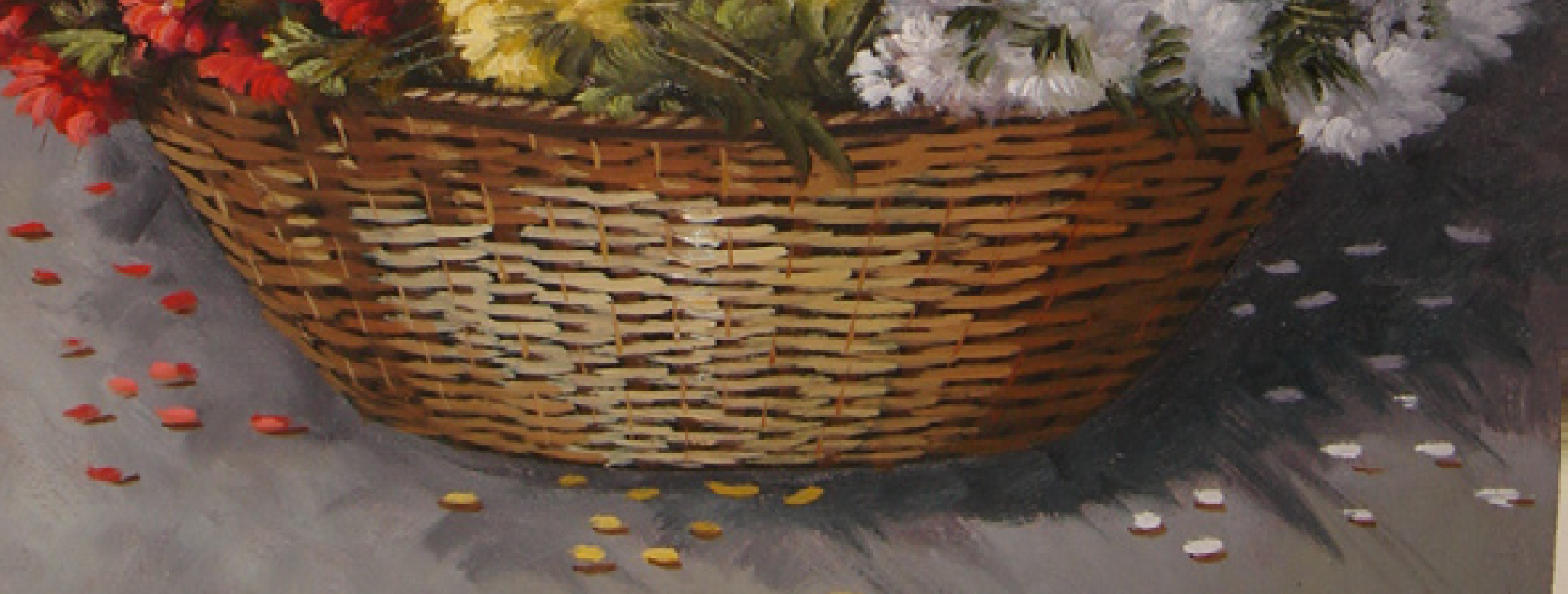




\title{
6 posibles impactos político culturales del CAFTA en los países centroamericanos. Comentarios a un estudio*
}

\author{
ROLANDO SIERRA FONSECA
}

El conocimiento sobre el pasado, el presente y el futuro sobre las sociedades centroamericanas se torna cada vez, no sólo necesario, sino urgente, sobre todo en un mundo como el actual caracterizado por fenómenos como la incertidumbre y la complejidad, que nos impiden ver con claridad el largo plazo. En buena medida un espacio importante en la producción sobre este conocimiento es el que viene procurando de generar el Programa Regional "Centroamérica en la Economía Mundial del Siglo XX”, con el patrocinio del IDRCN mediante la ejecución de una serie de proyectos y concursos de investigación en torno a generar, conocimientos, información y análisis de las posibilidades de la región de incorporarse al mundo globalizado de una manera distinta respecto de lo que han sido sus tendencias históricas de productor de materias primas o de mano de obra barata. En una serie de estudios patrocinados por este programa no es dable conocer las capacidades institucionales y económicas de la región en cuanto sus posibilidades comerciales, tecnológicas, productivas entre otras.

La presente jornada en que se realiza en nuestro país es ejemplo de ello al difundir y poner a la discusión los resultados de una serie de estudios sobre el impacto del CAFTA en la región y particularmente en Honduras. La importancia de esto radica no solo por la histórica carencia en el país de estudios que iluminen sobre esta temática, sino que los pocos que se han elaborado no han tenido la difusión debida a la comunidad intelectual y política de Honduras.

Un hecho importante a destacar dentro de este Programa Regional es que el estudio de la economía centroamericana se vincula a otras dimensiones como la social, la política y la cultural. No se puede olvidar que son escasas las obras que intentan explicar o comprender la región y sus países, ya que la complejidad del presente requiere de nuevos marcos y enfoques analíticos de largo alcance que en el presente resulta difícil de construir. Desde hace algún tiempo venimos moviéndonos con estudios de corto alcance, con estudios de caso o a lo sumo con bases de datos e información que no es posible comparar más que el pasado sobre la situación de los países de la región.

En esta perspectiva, a mi modo de ver, hay que ubicar el estudio: Impacto político y cultural del CAFTA en los países centroamericanos, CEHDES, realizado por Marvin Barahona, Ludwin Duarte y Suyapa Castro, el cual se trata de un trabajo de carácter aproximativo más que conclusivo sobre las posibles implicaciones políticas y culturales del CAFTA en la región.

Aunque, su conclusiones son parciales presentan algunos elementos para ir realizando análisis prospectivos del escenario centroamericano en el marco del CAFTA, a fin de ir buscando la síntesis de la dialéctica modernidad-modernización que acompañado a todas las propuestas de desarrollo socioeconómico de la región desde el siglo XIX, que han tenido serias implicaciones en las muchas experiencias

* Comentario a la Conferencia "Impacto Político y Cultural del CAFTA", Miércoles 26 de Enero de 2005, Tegucigalpa, Honduras, Organizada por el ASIES-IRDC fallidas o modernizaciones inconclusas, precisamente, por no asumir debidamente la cultura como una dimensión central del desarrollo.

La ausencia de la dimensión cultural en los procesos económicos parece repetirse en esta nueva etapa que significa la aprobación de una estrategia como el CAFTA, tal como se plantea el estudio bajo el siguiente enunciado: "los estados nacionales de la región le concedan un valor esencial al proceso de integración formal de sus economías con la de los Estado Unidos, sin adoptar medidas pertinentes para participar las consecuencias potenciales de tal hecho en el tejido socioeconómico, político y cultural de sus naciones respectivas"(p.2)

En este sentido, por medio de un sondeo sobre la percepción de los diferentes actores sociales centroamericano que representan a la sociedad civil, las instituciones corporativas de la empresa privada, partidos políticos, legislador, intelectuales y analistas relacionados con el tema, han estructurado la información obtenida en cinco capítulos:

El primero referido a los potenciales impactos políticos del CAFTA.

El segundo los potenciales impactos culturales.

El tercero intenta distinguir los efectos propios del CAFTA de aquello que habrían resultado de la globalización.

El cuarto contiene las conclusiones respecto a los impactos políticos y culturales, y el

Quinto presenta algunas medidas publicas y privadas para optimizar los beneficios del CAFTA o reducir sus perjuicios.

Las conclusiones de este trabajo apuntan, entre otras, a que los efectos políticos generales mas importantes del CAFTA, es el riesgo de fragmentación que corren la autonomía política y la soberanía que antes ejercían los estados nacionales. Al asumir compromisos como el de aceptar la jurisdicción de tribunales supranacionales para solucionar diferencias entre inversionistas y estados, o al aceptar la suscripción de un grupo de tratados adicionales para cumplir con lo estipulado por el CAFTA en materia de derechos de propiedad intelectual, los estados nacionales renuncian a una cuota de su autonomía y de sus soberanías tradicionales.

Este hecho implica, de acuerdo con los autores, la desterritorializaciòn del Estado Nacional y el inicio de un procesos de fragmentación del poder político y de la soberanía nacional, similar en sus rasgos al procesos de fragmentación que caracteriza el funcionamiento de la industria maquiladora" (54).

Otra conclusión en el campo de la cultura sobre un posible impacto del CAFTA es la acentuación del fenómeno de la transterritorialidad cultural como producto de la creciente migración de centroamericanos a Estados Unidos.

De acuerdo con los autores lo anterior, implica repensar las relaciones históricas y actuales con los Estados Unidos y revertir el antagonismo identidad-modernidad, para elaborar la cultura desde 
una visión del presente. Antagonismo, que a mi modo de ver, no puede leerse como tal, sino como una dialéctica orientada a producir una nueva síntesis.

Los comentarios a este trabajo se ubican en torno a tres ejes: metodológicos, conceptuales y de contenido.

Desde la perspectiva metodológica, tomando en cuenta que el objetivo del estudio es el de "identificar los impactos potenciales del CAFTA en la dimensión política y cultural de las sociedades centroamericanas, con el propósito de explicar su probable incidencia en las cinco naciones de área" me parece, que en el documento no se aclara si el objeto del mismo es el de realizar una cierta medición del impacto del CAFTA en "el tejido social, político y cultural de la republicas centroamericanas”, o un análisis prospectivo de los potenciales impactos.

Los instrumentos aplicados para la obtención de la información utilizados no están orientados a medir el posible impacto en estas áreas, ni a sistematizar experiencias o casos que alumbren sobre el tema. El estudio presenta la percepción sobre diversos personajes centroamericanos representativos de organizaciones e instituciones de los países de la región que dan su opinión sobre estos temas y con base en ello se presentan algunos temas relacionados con la política y la cultura.

Metodológicamente no se presenta el camino para visualizar de manera prospectiva los posibles impactos del CAFTA sobre la política y la cultura. Se carece de una matriz analítica para determinar cuáles son las posibles dimensiones e indicadores en estos campos que son más sensibles a ser afectos por la nueva dinámica económica que puede surgir a partir de implementación del CAFTA en la región. En este sentido, un estudio de esta naturaleza pudo haber sido una de punto de partida para establecer una línea de base sobre el estado político cultural de la región en una serie de indicadores. Es difícil percibir posibles impactos políticos culturales, sino se aísla adecuadamente la variable CAFTA, especialmente en un mundo como el actual caracterizado por la globalización, por lo tanto presionado por distintos mercados, relaciones comerciales, políticas y culturales.

Es importante tener claro que este es un estudio sobre la percepción de ciertos actores regionales que identifican problemas y posibles impactos políticos, culturales y sociales en la región. No se trata de un estudio de impacto, ya que no se definen variables e indicadores a evaluar. Como también se carece de una cierta línea de base desde donde poder comparar el impacto de un proceso como el CAFTA en la región. Creo que el estudio puede ser útil para ir definiendo una línea de base sobre el estado político y cultural de la región antes de la implementación del CAFTA.

Aun cuando en el estudio se buscan identificar algunos temas para observar el impacto político como el de la soberanía nacional y las reformas jurídico-institucionales y en el campo cultural se presentan las opiniones de los actores rurales y urbanos, los posibles impactos a la educación y especialmente a los derechos de propiedad intelectual y prestación de servicios y se esbozan las implicaciones que esto puede tener con la identidad nacional y cultural, sin embargo, me parece que se requiere establecer un marco más amplio en términos políticos y culturales sobre la región y hacerse algunas preguntas que orienten el proceso de investigación.

En primer lugar que el contexto político de la región se caracteriza por un proceso de transición a la democracia que en si misma tiene una serie de desafíos como la superación de una cultura autoritaria por una cultura política más participativa y representativa; cómo se legitima la democracia de origen y de desempeño, siendo que en la región conviven países, que de acuerdo con varios indicadores, que no han alcanzado un punto de no retorno a gobiernos no democráticos, en los estudios de opinión la preferencia por gobiernos no democráticos es creciente en algunos países y el abstencionismo electoral supera al partido ganador. Además que existe un alto grado de insatisfacción y desconfianza sobre las instituciones democráticas como puedan ser la justicia, los parlamentos y diputados, entre otras.

Por otra parte, la sociedad civil de la región se manifiesta cada vez más en oposición al CAFTA, lo cual significara un conflicto con los gobiernos nacionales en la medida que avance la aprobación del mismo. La pregunta, entonces, es que sin o con el CAFTA, se logrará un ambiente favorable para el fortalecimiento de las democracias, ya que uno de los principales desafíos que han tenido las democracias de la región es como se legitiman las economías de mercado frente a su poca capacidad de respuesta a los problemas de pobreza y desigualdad en la distribución de los ingresos.

Como lo han demostrado los estudios de Vop Rop y Lance Taylor, la liberalización en si misma no ha significado para muchos de los países de América Latina la disminución de la pobreza.

En este sentido, a nivel conceptual, en el estudio se hecha de menos, un concepto de cultura y más aún una discusión sobre cuál es el papel constituyente de la cultura en el desarrollo y la economía. Para Amartya Sen "Independientemente del concepto de desarrollo que adoptemos, la cultura tendrá un papel muy claro que desempeñar. Pero no es el mismo en ambos casos. En el concepto de opulencia, el papel de la cultura no sería fundamental (carece de valor intrínseco), sino puramente instrumental, es decir, puede ayudar a promover un acelerado crecimiento económico y aumentar la opulencia. No todos los sistemas de valores son igualmente eficaces en la promoción del crecimiento económico. Según varios expertos en ciencias sociales, ciertos sistemas de valores (como la ética protestante, o las prioridades confucianas) desempeñan un papel en el impulso de la industrialización y el crecimiento económico de Occidente, y más recientemente en el Oriente asiático. En este análisis y en este contexto, la cultura es algo que no se valora en sí mismo sino como un medio par alcanzar otros fines, en particular, los de promover y sostener la opulencia económica".

No puede haber duda de que este vínculo instrumental es de gran interés y relevancia, en virtud de que el proceso de crecimiento económico es por una razón u otro muy apreciado. Sin embargo, la pregunta que surge es: ¿Debe valorarse el crecimiento económico en sí mismo, llevando así al atesoramiento de esos elementos (incluyendo los parámetros culturales) que promueven el crecimiento? ¿O es el crecimiento económico en sí un instrumento y no puede reclamar un papel fundacional como pueden tenerlo los aspectos culturales de la vida humana? Es difícil pensar que la gente tiene buenas razones para valorar los bienes y los servicios, sin tomar en cuenta cómo afectan nuestra libertad de vivir en la forma en que la valoramos. También resulta difícil aceptar que el papel de la cultura puede ser plenamente capturado en un concepto puramente instrumental, Ciertamente, aquello que tenemos razón de valorar, nuestro tribunal de última instancia, debe estar relacionado con la cultura y, en este sentido, no podemos reducir la cultura a una posición secundaria como mero promotor del crecimiento económico. ¿Cómo podríamos hacer de nuestra valoración razonada algo absolutamente carente de valor?

Por tanto, es importante reconocer las funciones instrumentales de largo aliento de la cultura, en el proceso de desarrollo y, al mismo tiempo, reconocer que no todo es cultura en los juicios que se hacen sobre el desarrollo. Existe, además, un papel intrínseco en la evaluación del proceso de desarrollo. Este doble papel se aplica no sólo en el contexto de la promoción del desarrollo económico, sino a otros objetivos específicos externos, como la sustentabilidad del medio 
ambiente, la preservación de la diversidad de las especies, etc. En la promoción de todos esos objetivos específicos, algunos parámetros culturales pueden ser de ayuda y otros pueden ser un obstáculo. En tanto que tenemos razones para valorar estos objetivos específicos, tenemos bases derivadas e instrumentales para valorar esas posturas y características culturales que promueven el cumplimiento de dichos objetivos. Pero volvamos a la cuestión básica ¿por qué concentrarnos en estos objetivos específicos? La cultura debe ser considerada en grande, no como un simple medio para alcanzar ciertos fines, sino como su misma base social. No podemos entender la llamada dimensión cultural del desarrollo sin tomar nota de cada uno de estos papeles de la cultura.

La cultura, de acuerdo con Amartya Sen participa en el desarrolo en tres sentidos, distintos pero relacionados entre sí.

1. Papel constituyente: El desarrollo, en su sentido más amplio, incluye el desarrollo cultural, que es un componente básico e inseparable del desarrollo en general. Si se priva a las personas de la oportunidad de entender y cultivar su creatividad, eso es en sí un obstáculo para el desarrollo. Por tanto, la educación básica es importante no sólo por la contribución que puede hacer al crecimiento económico, sino porque es una parte esencial del desarrollo cultural.

2. Papel evaluativo: Lo que valoramos y que además tenemos razones para valorar está definitivamente influenciado por la cultura. El crecimiento económico o cualquier otro objetivo de esa clase, carecen de elementos externos importantes y las cosas que valoramos intrínsecamente, reflejan el impacto de nuestra cultura... Incluso si las mismas cosas tienen un alto valor en sociedades diferentes (si, por ejemplo, se busca vivir más tiempo y con mayor felicidad, en muchas sociedades muy diferentes), ello no las hace independientes de valores o de las culturas, sólo indica la congruencia de las distintas sociedades en sus razones para hacer tal valoración.

3. Papel instrumental: Independientemente de los objetivos que valoremos, su búsqueda estará influenciada, en mayor o menor grado, por la naturaleza de nuestra cultura y ética de comportamiento. El reconocimiento de este papel de la cultura es más frecuente que otros y si bien es cierto que no debemos limitarnos a este aspecto, no podemos ignorar el hecho de que los parámetros culturales desempeñan inter alia un fuerte papel instrumental. Esto se aplica no sólo a la promoción del crecimiento económico sino de otros cambios -como el mejoramiento en la calidad de vida- asociados con el desarrollo en un sentido amplio.

La libertad es primordial para la cultura, sobre todo, la libertad para decidir lo que habremos de valorar y qué clase de vida vamos buscar. En última instancia, el papel instrumental, el evaluativo y el constructivo están todos relacionados con esta libertad.

Una vez expuesto el planteamiento o la propuesta de esta obra haré algunos comentarios sobre los posibles impactos culturales y políticos del CAFTA en los países de la región es importante repensar la existencia de un cierto ethos cultural centroamericano, para desde ahí poder observar el papel que ha de jugar la cultura respecto al CAFTA, si es constituyente, evaluativo o instrumental.

Al contraponer el proyecto del CAFTA con la realidad política cultural centroamericana desde la perspectiva del desarrollo es tomar en cuenta que en Centroamérica existen diferentes visiones y regiones culturales. Como aparece en el primer Informe regional sobre Desarrollo Humano, sobresalen dos visiones la morazànica y la cartaga.

Sin embargo, la ilustración latinoamericana, de acuerdo con el sociólogo de la cultura el chileno Pedro Morandè, no fue un movimiento ni contra la corona (puesto que de ella venia), ni contra la nobleza (como en el caso europeo), sino contra los únicos sujetos de la síntesis cultural latinoamericana: el indio y el mestizo. La constitución de los estados nacionales vino a reforzar todavía más esta tendencia hasta transformar el criollismo a fines de siglo XIX en un fenómeno urbano comúnmente analizado por la sociología bajo el titulo: surgimiento y constitución de los sectores medios. En efecto, estos sectores son los herederos del proyecto cultural sincretista ideado por los jesuitas, continuado por los ilustrados laicistas y, desde el cuarenta y cinco, también por los neoiluminista cristianos y de todos los pelajes. Ocasionalmente estos sectores se definen a si mismos como "clase alta", pero este es más que un deseo de diferenciación social propio de la misma condición de la clase media crecida al amparo del Estado que, como se sabe, en América Latina no es un producto de la sociedad civil, sino su creador. La diferencia entre oligarquía y sectores medios es ciertamente de riqueza y de posición social, pero cualquiera que sea su magnitud no es lo suficiente como para ocultar su origen cultural común que, según Morandè, se identifica con el intento del criollo por desprenderse de su origen mestizo, sustituyendo la historia real del siglo XVI por un sincretismo universalista y sin historia.

Ahora bien, ¿se desprende de lo dicho que el criollo logró destruir la síntesis mestiza cristalizada ritualmente y sustituirla por una nueva síntesis cultural lograda esta vez en el plano de la palabra? La hipótesis puede ser, a este respecto, negativa. El intento de diferenciación del criollo ilustrado frente al mestizaje es ritualista en su esencia y solo puede someterse de espaldas a la historia real. Pero justamente este ritualismo es quien da la hegemonía a la síntesis cultural latinoamericana realizada por el mestizo, ya que su síntesis siempre fue ritual. Así, pese a las ideologías de moda y pese a los intentos modernizadores, el criollo ha permanecido atado a la legitimación cúltica y sacrificial del trabajo, verificando una y otra vez con su conducta el mismo ethos fundante que, por otra parte, se niega a reconocer como propio. Las síntesis culturales no se eligen a voluntad. Si son reales, ellas se imponen al conjunto de los individuos y grupos sin preguntar si les place. Pueden por cierto cambiarse, pero no negarse. Y si se desea cambiarla hay que partir por conocer su existencia y por descubrir empíricamente todas sus determinaciones. Pero esto es lo que las distintas teorías de la modernización desconocen. Piensan que para que exista sujeto histórico basta con sumar fuerzas a un plan de transformación definido idealmente. Piensan que se puede conceptuar toda la tradición histórica simplemente como lo no deseado, como lo que es 〈obstáculo〉 al desarrollo. Como esta es una operación imposible, el resultado no es otro que una profunda crisis de identidad y un reforzamiento de los mecanismos sacrificiales colectivos que pueden retrotraer la situación al momento de origen.

En este sentido, creo importante, analizar en los posibles impactos políticos y culturales del CAFTA, teniendo como marco las posibles interpretaciones de la cultura centroamericana como la existencia o no de un ethos cultural barroco, como lo interpreta Morande, o desde la perspectiva del espejo trizado de Joaquín Brunner, es decir que frente a la misma realidad los latinoamericanos nos vemos a nosotros mismos fragmentados. O probablemente el CAFTA profundice nuestra hibrides cultural de la cual nos habla Nestor García Canclini en la cual sigamos con nuestras artesanías y objetos pero producidos de manera homogénea con los colores y las formas que impone el consumo cultural de la globalización o quizás interpretarla bajo la lógica del sincretismo de un autor como Cristian Parker, en donde el ethos cultural centroamericano se funda en un nuevo ethos con los valores provenientes de los países del norte.

Entonces, lo importante creo actualmente es saber si la cultura se convertirá en una dimensión que facilitara el proyecto del CAFTA o estará ahí su mayor resistencia, más que en la política. 